\title{
Neurobehavioral Disorder Associated with Prenatal Alcohol Exposure (ND-PAE): Review of Evidence and Guidelines for Assessment
}

\author{
Lauren R. Doyle ${ }^{1} \cdot$ Sarah N. Mattson ${ }^{1}$
}

Published online: 27 June 2015

(C) Springer International Publishing Switzerland 2015

\begin{abstract}
The effects of prenatal alcohol use have been well documented. In this review, we discuss the inclusion of Neurobehavioral Disorder Associated with Prenatal Alcohol Exposure (ND-PAE) as a condition for further study in the $D i$ agnostic and Statistical Manual of Mental Disorders-Fifth Edition (DSM-5). We present a review of the evidence for impairment in three domains highlighted in ND-PAE: neurocognitive functioning, self-regulation, and adaptive functioning. In addition, we provide guidelines for clinical assessment of each domain. When considering ND-PAE, it is essential to obtain as comprehensive an assessment as possible, including multidisciplinary/multimethod assessment of the individual by a qualified team. It is our aim to provide clinicians with a useful reference for assessing ND-PAE and highlight important guidelines to be followed when conducting neuropsychological assessment.
\end{abstract}

Keywords Fetal alcohol syndrome (FAS) · Fetal alcohol spectrum disorders $\cdot$ Diagnosis $\cdot$ Prenatal alcohol exposure . Neuropsychological testing $\cdot$ Assessment

\section{Introduction}

Prenatal alcohol exposure (PAE) can have a myriad of physical, cognitive, and behavioral effects on the developing fetus

This article is part of the Topical Collection on Fetal Alcohol Syndrome

Sarah N. Mattson

sarah.mattson@sdsu.edu

1 Center for Behavioral Teratology, San Diego State University, 6330 Alvarado Court, Suite 100, San Diego, CA 92120, USA
$[1,2,3 \cdot, 4]$ that are encompassed within fetal alcohol spectrum disorders (FASDs) [3•]. Diagnoses within the FASDs include fetal alcohol syndrome (FAS), partial FAS, alcoholrelated neurodevelopmental disorder (ARND), and alcoholrelated birth defects (ARBD). Both FAS and partial FAS require the presence of characteristic facial dysmorphology [4].

While the diagnoses of FAS and partial FAS are based primarily on physical features, the most significant and impactful effects related to PAE are those on the central nervous system that manifest as cognitive and behavioral deficits. Such effects are noted as part of ARND, as "marked impairment in the performance of complex tasks" in children with normal growth and structural development [4] but are not well defined. These cognitive and behavioral effects are addressed further in the Diagnostic and Statistical Manual of Mental Disorders-Fifth Edition (DSM-5) [5•]. Neurobehavioral Disorder Associated with Prenatal Alcohol Exposure (ND-PAE) is included in the appendix of the DSM-5 under "conditions for further study." ND-PAE encompasses the range of neurobehavioral effects seen following PAE and can occur in children with histories of PAE independently of any physical effects (i.e., an exposed child with or without FAS can meet diagnostic criteria for ND-PAE). The DSM-5 provides detailed diagnostic criteria (Fig. 1). In addition to a history of more than minimal PAE, the proposed criteria for ND-PAE require impairment in three functional domains: neurocognitive, self-regulation, and adaptive functioning.

In this paper, we review the evidence for each of the diagnostic domains of ND-PAE and provide guidelines for assessment of each domain. It should be noted that these three domains are not the only effects of PAE, but are reviewed herein in the context of ND-PAE. For all neuropsychological assessment, standardized procedures should be followed. This includes administration and interpretation by trained and qualified individuals, and use of measures that are reliable, valid, 
Fig. 1 Criteria for Neurobehavioral Disorder Associated with Prenatal Alcohol Exposure (ND-PAE) from the Diagnostic and Statistical Manual of Mental DisordersFifth Edition (DSM-5) [5•]

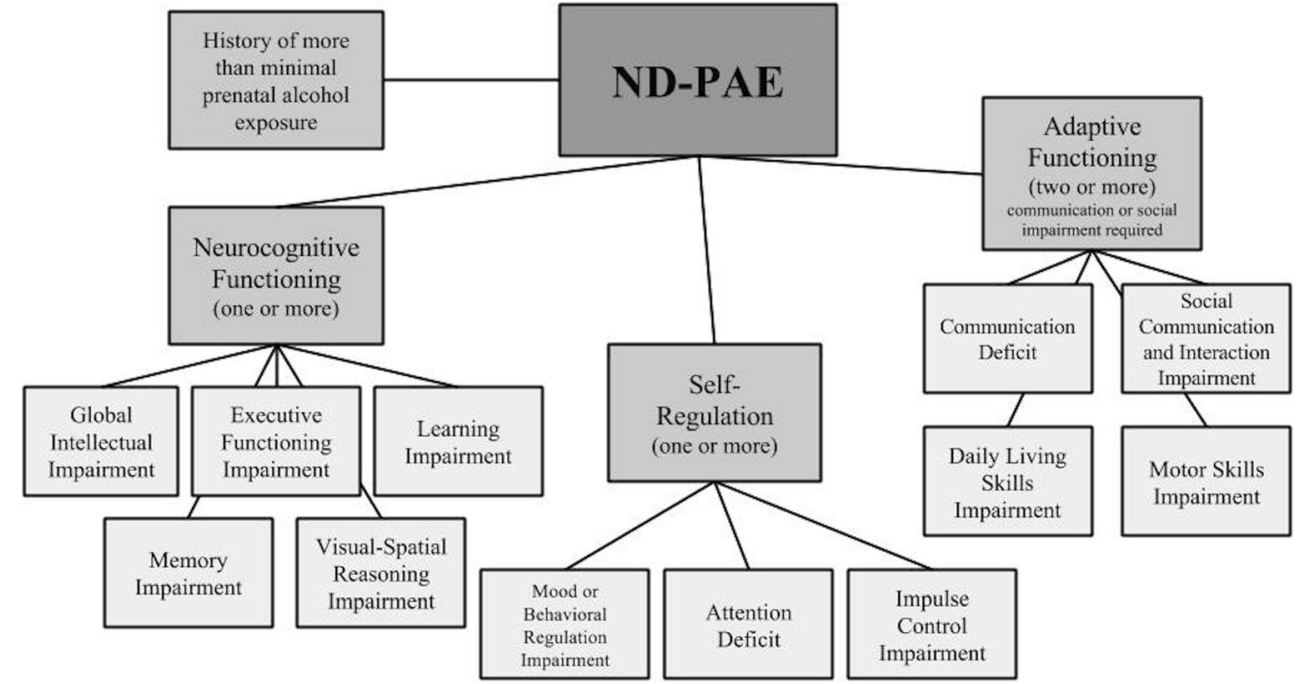

age- and culture-appropriate, and current. Tests included herein are current as of this writing; however, tests are frequently updated and the most current versions should be substituted, where appropriate. Furthermore, tests with normative data similar to the background of the patient should be used. When evaluating test performance, standardized scores should be used (e.g., $T$ scores, scaled scores) whenever possible. Comparison of an individual's test score on a given measure to normative data indicates how much that score differs from the population average and, when compared to other test results, can provide an estimate of relative impairment or strength in a given area. One question that remains to be determined empirically is what cutoff to use to indicate impairment [6-9], though ongoing research suggests that a 1 standard deviation (SD) cutoff is optimal in terms of sensitivity and specificity of ND-PAE $[10,11]$. The proposed guidelines for ND-PAE do not specify a required level of impairment, and thus clinical judgment is required. The guidelines also indicate onset of ND-PAE in childhood. As such, we focus this review on assessment of school-age children. However, suggestions for younger and older individuals are provided. Please see Moore and Riley, 2015 in this issue [12] for discussion of the need for more research in adults with FASDs or ND-PAE.

\section{Neurocognitive Impairment}

To meet the requirement of neurocognitive impairment, a child must display deficits in at least one of the following: (1) global IQ (defined as 70 or below on a standard IQ test), (2) executive functioning, (3) learning, (4) memory, and (5) visual spatial reasoning.

\section{Global IQ}

Deficits in overall IQ are probably the best documented of the effects of PAE [13]. Average IQ scores of alcohol-exposed children are typically in the low average to borderline ranges and children of mothers who drank throughout pregnancy have significantly lower IQ scores than children of mothers who never drank [14]. While more severe impairment is frequently seen in accordance with greater physical dysmorphology [15], this deficit in global IQ is not limited to alcohol-exposed children with physical dysmorphology [16].

While an IQ score in the intellectual deficient range $(<70)$ does not occur in all children with histories of PAE, and as such is not required for ND-PAE, its presence indicates neurocognitive impairment. The ND-PAE criteria specify global IQ deficit as an "IQ of 70 or less, or a standard score of 70 or below on a comprehensive developmental assessment" [5•]. There are several ways in which one can clinically assess for global IQ impairment. One option is to use the Wechsler Intelligence Scales for Children-Fifth Edition (WISC-V) [17]. The WISC-V is a measure of general intelligence for children 6 to 16 years old which provides an overall, full scale IQ (FSIQ) score, as well as five index scores indicating performance on measures of verbal ability, processing speed, working memory, visual spatial ability, and fluid reasoning. The FSIQ score obtained from the WISC-V provides an estimate of the child's general cognitive ability. It has a relatively short administration time of approximately 65 $80 \mathrm{~min}$. Similar assessment methods and scores are available for younger (ages 2.5-7.5 years) and older (16 years and older) individuals using the Wechsler Preschool and Primary Scale of Intelligence-Fourth Edition (WPPSI-IV) [18] and the Wechsler Adult Intelligence Scale-Fourth Edition (WAIS-IV) 
[19], respectively. If time is an issue, the Wechsler Abbreviated Scale of Intelligence-Second Edition (WASI-II) [20] also provides an estimate of IQ for individuals aged 6 to 80 years, and only takes about $30 \mathrm{~min}$ to administer. However, the WASI should only be used to identify whether more indepth testing is required.

Other measures that provide an estimate of overall ability include the Differential Ability Scales-Second Edition (DAS-II) [21], which calculates a global functioning score (the General Conceptual Ability or GCA score) for children aged 2.5 to 17 years old, similar to the FSIQ from the WISC-V. The authors of the DAS-II distinguish GCA from IQ by highlighting that the term IQ carries undue weight in the general population, and as such have identified a concept that captures general cognitive ability without the connotation of "IQ." They argue GCA provides a more homogeneous and pure estimate of true cognitive ability by only including subtests that load strongly onto this construct (i.e., verbal, visual-spatial, and reasoning tasks) [21]. In general, however, the GCA can be used as an IQ estimate. Administration time of the DAS-II is about 45-60 min. Two other tests of general ability should be mentioned. The Stanford-Binet Intelligence Scales, Fifth Edition (SB5) [22], can be used with individuals ages 2 to 85 and above and is particularly useful in lower functioning individuals. The Leiter International Performance Scale, Third Edition (Leiter-3) [23] is a measure for assessing nonverbal intelligence (ages 3 to 75 years and above) and is useful for nonverbal individuals. All these measures of global functioning provide standard scores (mean $=100, \mathrm{SD}=15$ ); a score of 70 or less on any one test is indicative of impaired global IQ.

If direct assessment of IQ is not possible, the Child and Adolescent Intellectual Disability Screening Questionnaire (CAIDS-Q) [24] may also be used to estimate a child's global intellectual functioning. The CAIDS-Q is a reliable and valid self- or caregiver-report questionnaire for use with individuals aged 8 to 18 and can help identify children with an intellectual disability. It provides a total percentage score, which can then be compared to a cut-off score to determine whether intellectual disability is probable. However, this measure should be used only as a screening tool to identify whether more indepth testing is required.

\section{Executive Functioning Impairment}

Several studies have highlighted the deficits in executive functioning associated with PAE [25•, 26-29]. Direct neuropsychological assessment indicates the greatest deficits in cognitive flexibility/set shifting, abstraction, spatial working memory, and verbal fluency [30] and parent reports indicate greatest impairment in inhibitory control and problemsolving [31].
Deficits in executive functioning may manifest as poor planning and organization, inflexibility, or difficulty with behavioral inhibition [5•]. Measures for assessing executive functioning impairment include the Delis-Kaplan Executive Function System (D-KEFS) [32] and the Behavior Rating Inventory of Executive Function (BRIEF) [33]. The DKEFS assesses executive functioning abilities for individuals aged 8 to 89 years old. It has nine stand-alone tests that each provides an estimate of overall executive functioning ability in the form of scaled scores (mean $=10, \mathrm{SD}=3$ ). A score of $1 \mathrm{SD}$ or more below the mean is indicative of executive functioning impairment. The BRIEF is a parent- or caregiver-report questionnaire for children aged 5 to 18 years. It provides the global executive composite $T$ score $($ mean $=50, \mathrm{SD}=10)$. Scores above 65 are indicative of clinical impairment in executive functioning.

\section{Learning Impairment}

It has been well documented that children with PAE experience greater academic problems than non-exposed children. Notably, children with PAE have shown impairment in math and reading [13, 34-36], which may lead to a specific learning disability [14]. These academic deficits persist after controlling for IQ [37]. In addition to academic performance, children with PAE have poor learning when asked to recall complex information [38] or more simple verbal and non-verbal information $[39,40]$. In particular, memory deficits appear to be due to disproportionate deficits in the encoding or learning of information rather than in recall, at least for verbal information $[39,40]$.

Learning deficits may be evident if the child has lower academic achievement than expected for their intellectual level or a diagnosis of specific learning disability [5•]. Methods to assess for learning impairment include the Woodcock-Johnson, Fourth Edition (WJ-IV) Tests of Achievement [41], a measure of academic achievement. The WJ-IV provides a comprehensive assessment of learning abilities for children and adults ages 2 to 90 . This measure provides standard scores $($ mean $=100, \mathrm{SD}=15)$ as well as age and grade equivalents. Other options for measuring learning impairment are the Wide Range Achievement Test-4 (WRAT-4) [42] for ages 5 to 94 and the Wechsler Individual Achievement Test-Third Edition (WIAT-III) [43] for ages 4 to 50. Both tests provide standard scores (mean $=100, \mathrm{SD}=15$ ) and the WRAT-4 also provides grade equivalents. The WRAT-4 should only be used as a screening test to determine whether more in-depth assessment is needed [44].

Additional measures include the California Verbal Learning Test, Children's version (CVLT-C) [45], a measure of verbal learning and memory for children ages 5 to 16 . The CVLT$\mathrm{C}$ provides a $T$ score $($ mean $=50, \mathrm{SD}=10)$ for overall learning ability and the ability to examine learning independently from 
more protracted memory. Indices on the CVLT-C that provide a more direct examination of a child's learning ability include List A Trials 1-5, List A Trial 5, learning slope, and percentage recall consistency. All indices provide a $z$ score (mean $=0$, $\mathrm{SD}=1$ ) except the index of List A Trials $1-5$, which provides a $T$ score. For adolescents and adults 16 to 89 years old, the California Verbal Learning Test, Second Edition (CVLT-II) [46] is available and provides similar scores as the CVLT-C. A score of 1 SD or more below the mean on any of the indices highlighted above is indicative of a learning impairment.

\section{Memory Impairment}

Children with PAE have deficits in memory including auditory memory, memory for stories and designs, and spatial memory [25•]. Deficits persist even when mental age is controlled or subjects are matched on IQ [39, 47]. Memory deficits are pervasive, occurring for both verbal and non-verbal material and are demonstrated using both free recall and recognition (forced choice or multiple choice) testing. As indicated above, memory deficits may stem from difficulty encoding new information and while retention of learned material is on par with matched controls [39, 48-50], it is important to note that clinical evaluation of learning and memory are confounded and the child will experience learning deficits as difficulty with memory.

Memory impairment may manifest as problems remembering information learned previously, trouble remembering lengthy verbal instructions, or repeatedly making the same mistakes [5•]. Assessment methods to evaluate memory impairment include the CVLT-C and CVLT-II, described earlier. Indices that provide more direct examination of memory impairment include the Short- and Long-Delay indices (both Free- and Cued-Recall), as well as the Recognition index. These indices provide a $z$ score $($ mean $=0, \mathrm{SD}=1)$. Importantly, performance on these measures should be considered in light of performance on the learning measures, described above. Similar levels of performance on learning and recall trials are indicative of encoding rather than retrieval deficits, while disproportionately low scores on recall trials indicate additional memory deficits. CVLT-C savings scores may also be useful. Other measures include memory subtests from the NEPSY-II [51]. The NEPSY-II assesses several domains including memory and learning, in children ages 3 to 16 , and provides scaled scores (mean $=10, \mathrm{SD}=3$ ). The Wide Range Assessment of Memory and Learning, Second Edition (WRAML-2) [52] is another measure that assesses memory for individuals aged 5 to 90 years old, and provides index standard scores (mean $=100, \mathrm{SD}=15)$ as well as subtest scaled scores (mean $=10, \mathrm{SD}=3$ ). Other options for measuring memory impairment include the Hopkins Verbal Learning TestRevised (HVLT-R) [53] for individuals aged 16 to 92 years, which provides $T$ scores (mean $=50, \mathrm{SD}=10)$ and the
Wechsler Memory Scale-Fourth Edition (WMS-IV) [54] for individuals ages 16 to 90 , which provides standard scores (mean $=100, \mathrm{SD}=15)$. A score of $1 \mathrm{SD}$ or more below the mean is indicative of memory impairment.

\section{Visual-Spatial Reasoning Impairment}

Although less well studied, children with PAE have deficits on visual-spatial construction tasks $[13,55-58]$ as well as visualspatial reasoning tasks [59]. One study showed that children with PAE process visual information differently than their non-exposed counterparts. On a task of hierarchical processing, large symbols (the global feature) were made up of smaller symbols (the local feature). In this study, children with PAE had greater difficulty processing the local features than typically developing controls [60].

Impairment in visual-spatial reasoning can manifest in disorganized or poorly planned drawings or constructions and problems differentiating left from right [5•]. Options for assessing visual-spatial reasoning include the visuospatial processing subtests from the NEPSY-II [51] and the BeeryBuktenica Developmental Test of Visual Motor Integration (VMI) [61]. The NEPSY-II has multiple measures of visual spatial function, including the Arrows, Geometric Puzzles, and Picture Puzzles subtests. The VMI is a method for assessing for visual-motor deficits for individuals 2 to 99 and provides a standard score (mean $=100, \mathrm{SD}=15$ ). The VMI provides a single score for visual-spatial ability and is recommended over the NEPSY-II Design Copying subtest. A score of $1 \mathrm{SD}$ or more below the mean on any of these measures is indicative of visual-spatial impairment.

\section{Self-Regulation Impairment}

To meet the requirement of impairment in self-regulation, a child must display deficits in at least one of the following: (1) mood or behavioral regulation, (2) attention, and (3) impulse control.

\section{Mood or Behavioral Regulation Impairment}

Children with PAE are at increased risk of developing major depressive disorder and have higher rates of negative affect [62-64]. Furthermore, children with PAE have increased rates of externalizing and internalizing problems [29]. Rates of oppositional defiant disorder and conduct disorder are also elevated in alcohol-exposed children [62, 65-69], further supporting deficits in mood and behavioral regulation.

Ways in which mood or behavioral regulation impairment may manifest include negative affect or irritability, frequent behavioral outbursts, or mood lability [5•]. Comprehensive methods of assessing these impairments are the Computerized 
Diagnostic Interview-Fourth Edition (C-DISC-IV) [70] for children ages 6 to 17 and the Schedule for Affective Disorders and Schizophrenia-School Age (K-SADS-PL) [71] for children ages 6 to 18. Both of these interviews assess the presence of psychiatric disorders or subsyndromal symptoms in a structured or semi-structured manner. Since the administration times for these assessments can be over an hour and require considerable training, parent- or self-report measures such as the Child Behavior Checklist (CBCL) [72] or Behavior Assessment System for Children-Second Edition (BASC-2) [73] are clinically appropriate alternatives. The CBCL can be used for children aged 6 to 18 . It provides $T$ scores (mean $=50, \mathrm{SD}=$ 10) for 8 problem behavior scales and 6 DSM-oriented scales. Scores between 65 and 70 indicate borderline impairment, and scores above 70 indicate clinical impairment. The Youth Self Report (YSR) [72] is also available for children aged 11 to 18 and provides similar scores as the CBCL. The BASC-2 can be used for children 2 to 5 years old (preschool form), children 6 to 11 years old (child form), and adolescents 12 to 21 years old (adolescent form). The BASC-2 provides $T$ scores (mean $=50$, $\mathrm{SD}=10$ ). On the externalizing, internalizing, and behavioral symptoms domains, $T$ scores between 60 and 70 indicate elevated impairment, while $T$ scores above 70 indicate clinical impairment.

\section{Attention Deficit}

Numerous studies have highlighted the presence of attention deficits among children prenatally exposed to alcohol [13, 14, 34, 38, 62, 74-77]. Importantly, children with PAE have higher rates of attention-deficit/hyperactivity disorder (ADHD) diagnoses than typically developing children [62, $65,66,78]$. In addition to clinical diagnoses, attention deficits comprise difficulty investing, organizing, and maintaining attention, and inhibiting impulsive responses [77]. Furthermore, children with PAE show attention deficits on measures of information processing, vigilance, and reaction time [38, $74-76,79,80]$. Ability to inhibit responses as well as variable reaction times on computerized vigilance tasks are particularly salient following PAE [38]. Behaviorally, teacher [34, 81, 82, $26]$ and parent [83-85] reports frequently highlight attention difficulties in children with PAE.

Attention deficits may manifest as difficulty shifting attention or difficulty sustaining mental effort [5•]. Several methods for assessing attention exist. Computerized vigilance tasks include Conners' Continuous Performance Test, Third Edition (CPT-III) [86], the Test of Variables of Attention (TOVA) [87], and the Quotient ADHD System [88]. The CPT-III can be used for children 8 years and above, and measures a variety of aspects of inattention. $T$ scores (mean $=50$, $\mathrm{SD}=10$ ) for each domain are given. The TOVA can be used for children and adults aged 4 to 80 years and above and provides an attention performance index score that suggests whether the individual's performance was within normal limits or is instead indicative of an attention deficit in addition to scales of inattention and impulsivity presented as standard scores (mean $=100, \mathrm{SD}=15$ ). The Quotient ADHD System can be used for children aged 6 to 55 and provides an index of symptom severity as well as composite scaled scores (scaled from $0-10$, with scores closer to 10 more indicative of ADHD). In addition, several aspects of attention are measured, and are presented as percentiles (percentiles below 16 indicating a performance more indicative of ADHD).

Behavioral reports (e.g., CBCL, YSR, BASC-2) can also provide an indication of whether an attention deficit is present. Often, behaviors such as these become apparent in the classroom, where a child spends the majority of the day, and as such, teachers can be a good resource. Elevation on the Attention Problems or Attention-Deficit/Hyperactivity Problems scales of the CBCL or YSR of 1 SD or more may indicate an attention deficit. Elevation on the Attention Problems scale of the BASC-2 may also indicate an attention deficit. Other measures available to assess attention deficit include the Conners Third Edition (Conners 3) [89]. The Conners 3 is available in parent, teacher, and youth report versions for children aged 6 to 18 . $T$ scores $($ mean $=50, \mathrm{SD}=10)$ are provided for 13 problem behavior scales. Elevation of $1 \mathrm{SD}$ or more on any ADHD scales of the Conners 3 may indicate presence of an attention deficit.

\section{Impulse Control Impairment}

Children with PAE have an increase in impulsive responses [13]. One study showed that teachers of children with PAE have been more likely to endorse an item labeled "Can't Wait Turn" on a measure assessing behavior in class [34]. Children with FASDs also have significantly higher externalizing behaviors than children with ADHD [90]. As has been previously stated, rates of oppositional defiant disorder and conduct disorder are higher among children with PAE [62, 65-68], which may suggest difficulty following rules or controlling impulsive behaviors.

Deficits in impulse control may manifest as difficulty waiting one's turn or difficulty complying with rules [5•]. Methods for assessment are similar to those for mood or behavioral regulation impairment (i.e., the C-DISC-IV, KSADS-PL, CBCL, YSR, BASC-2), but additional measures include teacher-report questionnaires, such as the CBCL or BASC-2 teacher versions. Scores above 70 on the parent or teacher versions of the CBCL are indicative of clinical impairment in impulse control, while scores between 65 and 70 indicate borderline impairment. On the parent or teacher version of the BASC-2, $T$ scores between 60 and 70 indicate borderline impairment, while scores above 70 indicate clinical impairment. Other measures available to assess impulse 
control include the Conners 3. Scores of 1 SD or more above the mean indicate impairment in impulse control.

\section{Adaptive Functioning Impairment}

To meet the requirement of impairment in adaptive functioning, a child must display deficits in at least two of the following (with at least one of (1) or (2) present): (1) communication deficit, (2) social impairment, (3) impairment in daily living, and (4) motor impairment. Of note is ongoing work examining whether requiring impairment in only the adaptive functioning domain improves sensitivity and specificity of NDPAE criteria $[10,11]$.

\section{Communication Deficit}

Language disorders among children with PAE are not well studied. However, deficits in aspects of language and verbal abilities in general have been well documented [13]. Common language impairments associated with PAE include word comprehension, naming ability, and expressive and receptive skills $[13,56,91]$. Additional language deficits include phonological processing deficits and speech production and articulation errors [92, 93]. Deficits are also noted on the communication domain of the VABS-II and its predecessor (VABS) [94], which include questions about expressive, receptive, and written language $[15,95,96]$. Importantly, in one study, no subjects with PAE obtained age-appropriate scores on the VABS Communication domain [15]. Further, as compared to children with ADHD, children with FASDs have significantly greater deficits on the WISC-IV Verbal Comprehension Index (VCI), which requires verbal responses [90].

Deficits in communication may become evident in everyday life as delayed acquisition of language or difficulty understanding spoken language [5•]. Both direct assessment of language function as well as parent report can be used to assess this domain. Direct measures of language and communication include the Comprehensive Receptive and Expressive Vocabulary Test-Third Edition (CREVT-3) [97], the Clinical Evaluation of Language Fundamentals-Fifth Edition (CELF-5) [98], and the VCI of the WISC-V [17]. The CREVT-3 (ages 5 to 89) and the CELF-5 (ages 5 to 21) both measure receptive and expressive language skills. The Clinical Evaluation of Language Fundamentals-Preschool-Second Edition (CELF-Preschool-2) [99] is also available for children ages 3 to 6 and provides similar scores as the CELF-5. The VCI from the WISC-V (ages 6 to 16) measures expressive language skills. All these tests provide standard scores (mean $=100, \mathrm{SD}=15$ ) and scores of $1 \mathrm{SD}$ or more below the mean may indicate language impairment.

Parent report of communication ability is possible using the Vineland Adaptive Behaviors Scales, Second Edition (VABS-
II) Communication domain score [100] or the Conceptual domain score of the Adaptive Behavior Assessment System, Third Edition (ABAS-3) [101]. The VABS-II is a parent- or caregiver-report questionnaire for individuals up to the age of 90 that measures four domains of adaptive functioning, including Socialization, Daily Living Skills, and Motor Skills in addition to Communication. The ABAS-3 measures four domains (Conceptual, Practical, Social, and General) in individuals up to the age of 89 . Both measures provide results in the form of standard scores (mean $=100, \mathrm{SD}=15$ ) and scores of $1 \mathrm{SD}$ or more below the mean may indicate language impairment. When necessary and available, consultation with speech and language pathology professionals to comprehensively assess a child's communication abilities is preferable.

\section{Social Communication and Interaction Impairment}

Several studies have shown that children with PAE have difficulty in social interactions [102-104]. During social interactions, several different processes are occurring at once. Children with PAE struggle to balance the multitude of linguistic and social-cognitive tasks during social interactions and when responding, do not provide sufficient organization or information for listeners $[102,103]$. Often, children with PAE fail to take the viewpoint of the listener during interactions [104], which is an important component of social interactions. Parent ratings also indicate poor communication skills and social problems, even when IQ is controlled [83]. Children with PAE are also more likely to use ambiguous references and inappropriately distinguish concepts in narratives they formulated [105]. Importantly, impairment on the Socialization scale of the VABS-II may be the most severely affected domain in adaptive functioning for children with PAE $[15,95,106,107]$.

Examples of how social impairment can manifest include being overly friendly with strangers, difficulty understanding social consequences, or difficulty reading social cues [5•]. One option for assessing social skills is the CBCL, which provides $T$ scores (mean $=50, \mathrm{SD}=10$ ) for both social problems and social competence. A score between 65 and 70 on the Social Problems scale indicates borderline impairment, while a score above 70 indicates clinical impairment. On the Social Competence scale, a score between 30 and 35 indicates borderline impairment, while a score below 30 indicates clinical impairment. Both the VABS-II Socialization domain score and the ABAS-3 Social domain score are in the form of standard scores (mean $=100, \mathrm{SD}=15$ ) and a score of $1 \mathrm{SD}$ or more below the mean may indicate impairment. While elevated scores on these measures may also indicate social impairment associated with autism spectrum disorders (ASD), the Autism Diagnostic Interview-Revised (ADI-R) [108] may help to differentiate between the two disorders. Children with FASDs or ASD both show significantly elevated socially inappropriate behaviors on the ADI-R, but only 
Table 1 Neuropsychological assessments for ND-PAE domains with corresponding age range, population mean (M), and standard deviations (SD)

\begin{tabular}{|c|c|c|c|}
\hline & Neurocognitive functioning & Self-regulation & Adaptive functioning \\
\hline \multirow[t]{27}{*}{ Neuropsychological assessment } & Global intellectual performance & Mood/behavioral regulation & Communication \\
\hline & WISC-V (Ages 6-16; M=100, SD=15) & C-DISC-IV (Ages 6-17) & VCI of WISC-V (Ages 6-16: M=100, SD=15) \\
\hline & WPPSI-IV (Ages 2.5-7.5; M=100, SD=15) & K-SADS-PL (Ages 6-18) & CREVT-3 (Ages 5-89; M=100, SD=15) \\
\hline & WAIS-IV (Ages 16+; M=100, SD=15) & & CELF-5 (Ages 5-21; M=100, SD=15) \\
\hline & WASI-II (Ages 6-80; M=100, SD=15) & Attention deficit & CELF-Preschool-2 (Ages 3-6; M=100, SD=15) \\
\hline & DAS-II (Ages 2.5-17; M=100, SD=15) & CPT-III (Ages 8+; M=50, SD=10) & \\
\hline & SB5 (Ages 2-85+; M=100, SD=15) & TOVA (Ages 4-80; $\mathrm{M}=100, \mathrm{SD}=15$ ) & Motor \\
\hline & Leiter-3 (Ages 2-20; $M=100, S D=15$ ) & Quotient (Ages 6-55; M=10, SD=3) & NEPSY-II (Ages 3-16; M=10, SD=3) \\
\hline & & & VMI (Ages 2-99; M=100, SD=15) \\
\hline & Executive function & & PDMS-2 (Ages $0-5 ; \mathrm{M}=100, \mathrm{SD}=15$ ) \\
\hline & D-KEFS (Ages 8-89; M=10, SD=3) & & \\
\hline & & Impulse control & \\
\hline & & C-DISC-IV (Ages 6-17) & \\
\hline & & K-SADS-PL (Ages 6-18) & \\
\hline & Memory & & \\
\hline & CVLT-II (Ages 16-89; M=50, SD=10) & & \\
\hline & NEPSY-II (Ages 3-16; M=10, SD=3) & & \\
\hline & HVLT-R (Ages 16-92; M=50, SD=10) & & \\
\hline & WMS-IV (Ages 16-90; M=100, SD=15) & & \\
\hline & WRAML-2 (Ages 5-90; M=100, SD=15) & & \\
\hline & Learning & & \\
\hline & $\begin{array}{l}\text { WJ-IV (Ages 2-90M=100, SD=15; } \\
\text { age/grade equivalents) } \\
\text { WRAT-4 (Ages 5-94; } \mathrm{M}=100, \mathrm{SD}=15 \text {; } \\
\text { grade equivalents) } \\
\text { WIAT-III (Ages 4-50; } \mathrm{M}=100, \mathrm{SD}=15 \text { ) }\end{array}$ & & \\
\hline & CVLT-C (Ages 5-16; M=50, SD=10) & & \\
\hline & CVLT-II (Ages 16-89; M=50, SD=10) & & \\
\hline & Visual-spatial reasoning & & \\
\hline & Beery VMI (Ages 2-99; M=100, SD=15) & & \\
\hline & NEPSY-II (Ages 3-16; M=10, SD=3) & & \\
\hline \multirow[t]{10}{*}{ Behavioral report } & IQ estimate & $\begin{array}{l}\text { Mood/behavioral regulation, impulse } \\
\text { control, and attention deficit }\end{array}$ & Communication and daily living skills \\
\hline & CAIDS-Q (Ages 8-19; total percentage) & CBCL (Ages 6-18; M=50, SD=10) & VABS-II (Ages 0-90; M=100, SD=15) \\
\hline & & YSR (Ages 11-18; M=50, SD=10) & ABAS-3 (Ages $0-89 ; \mathrm{M}=100, \mathrm{SD}=15$ ) \\
\hline & Executive function & BASC-2 (Ages 2-21; M=50, SD=10) & \\
\hline & BRIEF (Ages 5-18; M=50, SD=10) & Conners 3 (Ages $6-18 ; \mathrm{M}=50, \mathrm{SD}=10$ ) & Social communication/interaction \\
\hline & & & CBCL (Ages 6-18; M=50, SD=10) \\
\hline & & & VABS-II (Ages 0-90; M=100, SD=15) \\
\hline & & & ABAS-3 (Ages $0-89 ; \mathrm{M}=100, \mathrm{SD}=15$ ) \\
\hline & & & Motor skills \\
\hline & & & VABS-II (Ages 0-90; M=100, SD=15) \\
\hline
\end{tabular}

WISC- $V$ Wechsler intelligence scale for children-fifth edition, WPPSI-IV Wechsler preschool and primary scale of intelligence-fourth edition; WAIS-IV Wechsler adult intelligence scale-fourth edition, WASI-III Wechsler abbreviated scale of intelligence-third edition, DAS-II Differential ability scales-second edition, SB5 Stanford Binet intelligence scales, fifth edition, Leiter-3 Leiter international performance scale, third edition, D-KEFS Delis-Kaplan executive function system, CVLT-C California verbal learning test-children's version, CVLT-II California verbal learning test-second edition, NEPSY-II NEPSY-second edition, HVLT-R Hopkins verbal learning test-revised, WMS-IV Wechsler memory scale-fourth edition, WRAML-2 Wide range assessment of memory and learning-second edition, WJ-IV Woodcock Johnson-fourth edition, WRAT-4 wide range achievement test-fourth edition, WIAT-III Wechsler individual achievement test-third edition, Beery VMI Beery-Buktenica developmental test of visual-motor integration, CAIDS- $Q$ child and adolescent intellectual disability screening questionnaire, BRIEF behavior rating inventory of executive function, $C-D I S C-I V$ computerized diagnostic interview schedule for children, $K$-SADS-PL schedule for affective disorders and schizophrenia for school-age children-present and lifetime version, CPT-III Conners' continuous performance test, third edition, TOVA test of variables of attention, Quotient quotient ADHD system, CBCL child behavior checklist, YSR youth self-report, $B A S C-2$ behavior assessment system for children-second edition, $V C I$ verbal comprehension index, CREVT-3 comprehensive receptive and expressive vocabulary test-third edition, $C E L F-5$ clinical evaluation of language fundamentals-fifth edition, PDMS-2 Peabody developmental motor scales-second edition, VABS-II Vineland adaptive behavior scales-second edition, $A B A S-3$ Adaptive behavior assessment system-third edition 
those with ASD have difficulties in other domains of the ADI$\mathrm{R}$, such as initiating social interaction [109]. As such, a profile marked by impairment in social communication and interaction as evaluated by the VABS-II, ABAS-3, or CBCL, while not displaying impairment on the ADI-R, may indicate ND-PAE.

\section{Daily Living Skills Impairment}

Children with PAE have deficits in performing daily living skills, an important aspect of adaptive function, and are less likely to be able to live independently $[15,106]$. In addition, children with PAE have greater deficits in daily living skills than children with ADHD [95]. In a study with an average subject age of 17 years, the average age equivalent of daily living skills was shown to be at the 9-year level for subjects with PAE [15]. Children with FASDs also perform significantly worse than children with a specific learning disability and controls across all domains of the VABS, including Daily Living Skills [110].

Impaired daily living skills can include delayed toileting, feeding, or bathing. Other examples of impaired daily living skills are difficulty managing daily schedule or problems following rules of personal safety [5•]. As is the case with other domains of adaptive functioning, the VABS-II is an excellent tool for measuring daily living skills impairment. Likewise, the ABAS-3 can be used to measure daily living skills. Both tests provide standard scores (mean $=100, \mathrm{SD}=15$ ) and scores of 1 SD or more below the mean on the Daily Living Skills domain of the VABS-II or the Practical domain of the ABAS3 is indicative of impairment.

\section{Motor Impairment}

Several studies have shown delayed motor development in infants and children with PAE [80, 111, 112]. These impairments include both gross and fine motor functions. In gross motor domains, children with FASDs have deficits in postural control [113]. Fine motor functions, such as hand-eye coordination, finger dexterity, and motor speed, are impaired bilaterally in children with PAE with and without FAS [114-116].

Motor impairment can manifest in several ways, including poor fine motor development, problems in coordination and balance, delayed attainment of gross motor milestones or even ongoing deficits in gross motor function [5•]. The Motor Skills domain of the VABS-II provides an estimate of motor functioning (ages 0 to 90 ) and impairment is indicated by a score of $1 \mathrm{SD}$ or more below the mean. Other methods for assessing motor impairment include the sensorimotor domain of the NEPSY-II and the Visual-Motor Integration Scale of the Beery VMI, which assesses visual-motor integration. As previously stated, the NEPSY-II provides scaled scores (mean= $10, \mathrm{SD}=3$ ) and the Beery VMI provides standard scores (mean $=100, \mathrm{SD}=15$ ). For children up to the age of 5 , the
Peabody Developmental Motor Scales, Second Edition (PDMS-2) [117] is available as a measure of gross and fine motor skills. The PDMS-2 provides standard scores (mean= $100, \mathrm{SD}=15$ ) as well as age equivalents for Fine, Gross, and Total Motor quotients. Scores on any measure of $1 \mathrm{SD}$ or more below the mean are indicative of motor impairment. For children age 5 and below, the PDMS- 2 is the preferred measure of motor skills. However, the VMI has the advantage of a wider age range. When necessary and available, consultation with physical or occupational therapy professionals is preferable to obtain as comprehensive an evaluation of a child's motor abilities as possible.

\section{Conclusion}

While we focused primarily on school-age children, it is important to note that difficulties do not start at school-entry and assessment at younger ages should be considered. However, some domains may not be readily assessed in younger children and fewer psychometrically sound assessments are available in this age range. Thus, while the assessment framework outlined could be used as a guide, attention needs to be paid to the reliability and validity of measurement tools.

From the multitude of studies presented herein, it is evident that children exposed to alcohol prenatally experience deficits in several areas of functioning. We have reviewed evidence for deficits in the three domains highlighted by the DSM-5 (neurocognitive functioning, self-regulation, and adaptive functioning) and ways in which these deficits may manifest in ND-PAE. We have also provided several options for clinically assessing the criteria within each domain (Table 1). This list is not exhaustive and care should be taken to select tests that are appropriate for use with the individual patient. It is important to note that while neuropsychological and behavioral assessment do provide a breadth of knowledge of a child's abilities and functioning, it is essential to obtain as comprehensive an evaluation as possible, emphasizing multidisciplinary assessments. In this way, the most complete and accurate profile can be created to determine an appropriate diagnosis and make essential interventions available.

Acknowledgments Preparation of this paper was supported by National Institute on Alcohol Abuse and Alcoholism grant U01 AA014834. Additional support was provided by T32 AA013525.

\section{Compliance with Ethics Guidelines}

Conflict of Interest Lauren R. Doyle and Sarah N. Mattson declare that they have no conflict of interest.

Human and Animal Rights and Informed Consent This article does not contain any studies with human or animal subjects performed by any of the authors. 


\section{References}

Papers of particular interest, published recently, have been highlighted as:

- Of importance

1. Jones KL, Smith DW, Ulleland CN, Streissguth AP. Pattern of malformation in offspring of chronic alcoholic mothers. Lancet. 1973;301(7815):1267-71. doi:10.1016/S0140-6736(73)91291-9.

2. Stratton K, Howe C, Battaglia F, editors. Fetal alcohol syndrome: diagnosis, epidemiology, prevention, and treatment. Washington: Institute of Medicine, National Academy Press; 1996.

3. Riley EP, Infante MA, Warren KR. Fetal alcohol spectrum disorders: an overview. Neuropsychol Rev. 2011;21(2):73-80. doi:10. 1007/s11065-011-9166-x. This paper reviews the diagnostic criteria for fetal alcohol spectrum disorders and provides a strong overview of the disorder.

4. Hoyme HE, May PA, Kalberg WO, Kodituwakku P, Gossage JP, Trujillo PM, et al. A practical clinical approach to diagnosis of fetal alcohol spectrum disorders: clarification of the 1996 Institute of Medicine criteria. Pediatrics. 2005;115(1):39-47. doi:10.1542/ peds.2004-0259.

5. American Psychiatric Association. Diagnostic and statistical manual of mental disorders, DSM-5. 5th ed. Arlington: American Psychiatric Publishing, Inc; 2013. This book is the comprehensive list of psychiatric conditions including Neurobehavioral Disorder (ND-PAE), see page 798.

6. Heaton RK, Grant I, Matthews CG. Comprehensive norms for an expanded Halstead-Reitan battery: demographic corrections, research findings, and clinical applications. Odessa: Psychological Assessment Resources; 1991.

7. Heaton RK, Miller SW, Taylor MJ, Grant I. Revised comprehensive norms for an expanded Halstead-Reitan battery: demographically adjusted neuropsychological norms for African American and Caucasian adults. Odessa: Psychological Assessment Resources; 2004.

8. Jak AJ, Bondi MW, Delano-Wood L, Wierenga C, CoreyBloom J, Salmon DP, et al. Quantification of five neuropsychological approaches to defining mild cognitive impairment. Am J Geriatr Psychiatry. 2009;17(5):368-75. doi:10. 1097/JGP.0b013e31819431d5.

9. Ganguli M, Blacker D, Blazer DG, Grant I, Jeste DV, Paulsen JS, et al. Classification of neurocognitive disorders in DSM-5: a work in progress. Am J Geriatr Psychiatry. 2011;19(3):205-10.

10. Mattson SN, Jones KL, Goh P, Sadler M, Riley EP, the CIFASD. Empirical evidence for sensitivity and specificity of the ND-PAE diagnosis. Manuscript submitted for publication. 2015.

11. Kable J, Coles C. Empirical evidence supporting the internal validity of the ND-PAE diagnosis. Manuscript submitted for publication. 2015.

12. Moore EM, Riley EP. What happens to children with fetal alcohol spectrum disorders when they become adults? Current Developmental Disorders Reports. 2015:in press.

13. Mattson SN, Riley EP. A review of the neurobehavioral deficits in children with fetal alcohol syndrome or prenatal exposure to alcohol. Alcohol Clin Exp Res. 1998;22(2):279-94. doi:10.1111/j. 1530-0277.1998.tb03651.x.

14. Coles CD, Brown RT, Smith IE, Platzman KA, Erickson S, Falek A. Effects of prenatal alcohol exposure at school age. I. Physical and cognitive development. Neurotoxicol Teratol. 1991;13(4):357-67.

15. Streissguth AP, Aase JM, Clarren SK, Randels SP, LaDue RA, Smith DF. Fetal alcohol syndrome in adolescents and adults. J Am Med Assoc. 1991;265(15):1961-7.
16. Mattson SN, Riley EP, Gramling L, Delis DC, Jones KL. Heavy prenatal alcohol exposure with or without physical features of fetal alcohol syndrome leads to IQ deficits. J Pediatr. 1997;131(5):71821. doi:10.1016/S0022-3476(97)70099-4.

17. Wechsler D. Wechsler intelligence scale for children-fifth edition (WISC-V). Pearson: Bloomington; 2015.

18. Wechsler D. Wechsler preschool and primary scale of intelligencefourth edition (WPPSI-IV). San Antonio: Psychological Corporation; 2012.

19. Wechsler D. Wechsler adult intelligence scale-fourth edition (WAIS-IV). San Antonio: Psychological Corporation; 2008.

20. Wechsler D. Wechsler abbreviated scale of intelligence, second Edition (WASI-II). San Antonio: Pearson; 2011.

21. Elliott CD. Differential ability scales - second edition (DAS-II). San Antonio: Harcourt Assessment; 2007.

22. Roid GH. Stanford Binet Intelligence Scale: Fifth Edition. Chicago: Riverside; 2003.

23. Roid GH, Miller LJ, Pomplun M. Leiter International Performance Scale-Third Edition (Leiter-3). Wood Dale: Stoelting Co; 2013.

24. McKenzie K, Paxton D, Murray G, Milanesi P, Murray AL. The evaluation of a screening tool for children with an intellectual disability: the child and adolescent intellectual disability screening questionnaire. Res Dev Disabil. 2012;33:1068-75. doi:10.1016/j. ridd.2012.01.015.

25. Mattson SN, Crocker N, Nguyen TT. Fetal alcohol spectrum disorders: neuropsychological and behavioral features. Neuropsychol Rev. 2011;21(2):81-101. doi:10.1007/s11065-011-9167-9. This paper provides a comprehensive review of the neurobehavioral features observed in individuals with fetal alcohol spectrum disorder.

26. Ware AL, Crocker N, O'Brien JW, Deweese BN, Roesch SC, Coles $\mathrm{CD}$, et al. Executive function predicts adaptive behavior in children with histories of heavy prenatal alcohol exposure and attention-deficit/hyperactivity disorder. Alcohol Clin Exp Res. 2012;36(8):1431-41. doi:10.1111/j.1530-0277.2011.01718.x.

27. Paolozza A, Rasmussen C, Pei J, Hanlon-Dearman A, Nikkel SM, Andrew G, et al. Deficits in response inhibition correlate with oculomotor control in children with fetal alcohol spectrum disorder and prenatal alcohol exposure. Behav Brain Res. 2014;259: 97-105. doi:10.1016/j.bbr.2013.10.040.

28. Rasmussen C, Tamana S, Baugh L, Andrew G, Tough S, Zwaigenbaum L. Neuropsychological impairments on the NEPSY-II among children with FASD. Child Neuropsychol. 2013;19(4):337-49. doi:10.1080/09297049.2012.658768.

29. Kodituwakku P, Kodituwakku E. Cognitive and behavioral profiles of children with fetal alcohol spectrum disorders. Curr Dev Disord Rep. 2014;1(3):149-60. doi:10.1007/s40474-014-0022-6.

30. Mattson SN, Roesch SC, Glass L, Deweese BN, Coles CD, Kable JA, et al. Further development of a neurobehavioral profile of fetal alcohol spectrum disorders. Alcohol Clin Exp Res. 2013;37(3): 517-28. doi:10.1111/j.1530-0277.2012.01952.x.

31. Nguyen TT, Glass L, Coles CD, Kable JA, May PA, Kalberg WO, et al. The clinical utility and specificity of parent report of executive function among children with prenatal alcohol exposure. J Int Neuropsychol Soc. 2014;20(7):704-16. doi:10.1017/ S1355617714000599.

32. Delis DC, Kaplan E, Kramer JH. The Delis-Kaplan Executive Function System: Examiner's Manual. San Antonio: The Psychological Corporation; 2001.

33. Gioia GA, Isquith PK, Guy SC, Kenworthy L. Behavior Rating Inventory of Executive Function: Professional Manual. Odessa: Psychological Assessment Resources, Inc; 2000.

34. Carmichael Olson H, Sampson PD, Barr H, Streissguth AP, Bookstein FL. Prenatal exposure to alcohol and school problems 
in late childhood: a longitudinal prospective study. Dev Psychopathol. 1992;4:341-59.

35. Howell KK, Lynch ME, Platzman KA, Smith GH, Coles CD. Prenatal alcohol exposure and ability, academic achievement, and school functioning in adolescence: a longitudinal follow-up. J Pediatr Psychol. 2006;31(1):116-26. doi:10.1093/jpepsy/jsj029.

36. Rasmussen C, Bisanz J. The relation between mathematics and working memory in young children with fetal alcohol spectrum disorders. J Spec Educ. 2011;45(3):184-91. doi:10.1177/ 0022466909356110.

37. Goldschmidt L, Richardson GA, Stoffer DS, Geva D, Day NL. Prenatal alcohol exposure and academic achievement at age six: a nonlinear fit. Alcohol Clin Exp Res. 1996;20(4):763-70.

38. Streissguth AP, Sampson PD, Olson HC, Bookstein FL, Barr HM, Scott M, et al. Maternal drinking during pregnancy: attention and short-term memory in 14-year-old offspring - a longitudinal prospective study. Alcohol Clin Exp Res. 1994;18(1):202-18. doi:10. 1111/j.1530-0277.1994.tb00904.x.

39. Mattson SN, Riley EP, Delis DC, Stern C, Jones KL. Verbal learning and memory in children with fetal alcohol syndrome. Alcohol Clin Exp Res. 1996;20(5):810-6. doi:10.1111/j.1530-0277.1996. tb05256.x.

40. Mattson SN, Roebuck TM. Acquisition and retention of verbal and nonverbal information in children with heavy prenatal alcohol exposure. Alcohol Clin Exp Res. 2002;26(6):875-82. doi:10. 1111/j.1530-0277.2002.tb02617.x.

41. Schrank FA, McGrew KS, Mather N. Woodcock-Johnson IV Tests of Achievement. Rolling Meadows: Riverside; 2014

42. Wilkinson GS, Robertson GJ. WRAT-4: Wide Range Achievement Test Professional Manual. Lutz: Psychological Assessment Resources, Inc; 2006.

43. Wechsler Individual Achievement Test-Third Edition (WIAT-III). Bloomington: Pearson; 2009.

44. Sattler JM. Assessment of children. San Diego: Jerome M. Sattler, Publisher, Inc.; 2001.

45. Delis DC, Kramer JH, Kaplan E, Ober BA. Manual for the California Verbal Learning Test-Children's Version. San Antonio: The Psychological Corporation; 1994.

46. Delis D, Kramer JH, Kaplan E, Ober BA. California Verbal Learning Test-Second Edition (CVLT-II) Manual. San Antonio: The Psychological Corporation; 2000.

47. Vaurio L, Riley EP, Mattson SN. Neuropsychological comparison of children with heavy prenatal alcohol exposure and an IQmatched comparison group. J Int Neuropsychol Soc. 2011;17(3): 463-73. doi:10.1017/S1355617711000063.

48. Crocker N, Vaurio L, Riley EP, Mattson SN. Comparison of verbal learning and memory in children with heavy prenatal alcohol exposure or attention-deficit/hyperactivity disorder. Alcohol Clin Exp Res. 2011;35(6):1114-21. doi:10.1111/j.1530-0277.2011.01444.x.

49. Kaemingk KL, Mulvaney S, Halverson PT. Learning following prenatal alcohol exposure: performance on verbal and visual multitrial tasks. Arch Clin Neuropsychol. 2003;18(1):33-47.

50. Willoughby KA, Sheard ED, Nash K, Rovet J. Effects of prenatal alcohol exposure on hippocampal volume, verbal learning, and verbal and spatial recall in late childhood. J Int Neuropsychol Soc. 2008;14(6):1022-33. doi:10.1017/S1355617708081368.

51. Korkman M, Kirk U, Kemp S. NEPSY-II. Administration Manual. 2nd ed. San Antonio: Harcourt Assessment; 2007.

52. Sheslow D, Adams W. Wide Range Assessment of Memory and Learning Second Edition (WRAML-2). Lutz: Psychological Assessment Resources; 2003.

53. Benedict RHB, Brandt J. Hopkins Verbal Learning Test-Revised (HVLT-R): Professional Manual. Lutz: Psychological Assessment Resources; 2001.

54. Wechsler D. Wechsler Memory Scale-Fourth Edition (WMS-IV). San Antonio: Pearson; 2009.
55. Aronson M, Hagberg B. Neuropsychological disorders in children exposed to alcohol during pregnancy: a follow-up study of 24 children to alcoholic mothers in Goteborg, Sweden. Alcohol Clin Exp Res. 1998;22(2):321-4.

56. Conry J. Neuropsychological deficits in fetal alcohol syndrome and fetal alcohol effects. Alcohol Clin Exp Res. 1990;14(5): 650-5. doi:10.1111/j.1530-0277.1990.tb01222.x.

57. Jirikowic T, Carmichael Olson H, Kartin D. Sensory processing, school performance, and adaptive behavior of young school-age children with fetal alcohol spectrum disorders. Phys Occup Ther Pediatr. 2008;28(2):117-36. doi:10.1080/01942630802031800.

58. Uecker A, Nadel L. Spatial locations gone awry: object and spatial memory deficits in children with fetal alcohol syndrome. Neuropsychologia. 1996;34(3):209-23.

59. Hunt E, Streissguth AP, Kerr B, Olson HC. Mothers alcohol consumption during pregnancy: effects on spatial-visual reasoning in 14-year-old children. Psychol Sci. 1995;6(6):339-42.

60. Mattson SN, Gramling L, Delis DC, Jones KL, Riley EP. Globallocal processing in children prenatally exposed to alcohol. Child Neuropsychol.1996;2(3):165-75. doi:10.1080/ 09297049608402249.

61. Beery KE, Beery NA. Manual for the Beery-Buktenica Developmental Test of Visual-Motor Integration. 5th ed. Minneapolis: NCS Pearson, Inc.; 2004.

62. Fryer SL, McGee CL, Matt GE, Mattson SN. Evaluation of psychopathological conditions in children with heavy prenatal alcohol exposure. Pediatrics. 2007;119(3):E733-41. doi:10.1542/peds.2006-1606.

63. O'Connor MJ. Prenatal alcohol exposure and infant negative affect as precursors of depressive features in children. IMHJ. 2001;22(3):291-9.

64. O'Connor MJ, Paley B. The relationship of prenatal alcohol exposure and the postnatal environment to child depressive symptoms. J Pediatr Psychol. 2006;31(1):50-64.

65. Burd L, Klug MG, Martsolf JT, Kerbeshian J. Fetal alcohol syndrome: neuropsychiatric phenomics. Neurotoxicol Teratol. 2003;25(6):697-705. doi:10.1016/j.ntt.2003.07.014.

66. D'Onofrio BM, Van Hulle CA, Waldman ID, Rodgers JL, Rathouz PJ, Lahey BB. Causal inferences regarding prenatal alcohol exposure and childhood externalizing problems. Arch Gen Psychiatry. 2007;64(11):1296-304. doi:10.1001/archpsyc.64.11.1296.

67. Disney ER, Iacono W, McGue M, Tully E, Legrand L. Strengthening the case: prenatal alcohol exposure is associated with increased risk for conduct disorder. Pediatrics. 2008;122(6):e1225-30. doi:10.1542/peds.2008-1380.

68. Steinhausen H-C, Spohr H-L. Long-term outcome of children with fetal alcohol syndrome: psychopathology, behavior and intelligence. Alcohol Clin Exp Res. 1998;22(2):334-8.

69. Ware AL, O'Brien JW, Crocker N, Deweese BN, Roesch SC, Coles $\mathrm{CD}$, et al. The effects of prenatal alcohol exposure and attention-deficit/hyperactivity disorder on psychopathology and behavior. Alcohol Clin Exp Res. 2013;37(3):507-16. doi:10. 1111/j.1530-0277.2012.01953.x.

70. Shaffer D, Fisher P, Lucas CP, Dulcan MK, Schwab-Stone ME. NIMH Diagnostic Interview Schedule for Children Version IV (NIMH DISC-IV): description, differences from previous versions, and reliability of some common diagnoses. J Am Acad Child Adolesc Psychiatry. 2000;39(1):28-38. doi:10.1097/ 00004583-200001000-00014.

71. Kaufman J, Birmaher B, Brent D, Rao U, Flynn C, Moreci P, et al. Schedule for Affective Disorders and Schizophrenia for SchoolAge Children - Present and Lifetime Version (K-SADS-PL): initial reliability and validity data. J Am Acad Child Adolesc Psychiatry. 1997;36(7):980-8.

72. Achenbach TM, Rescorla LA. Manual for the ASEBA SchoolAge Forms \& Profiles. Burlington: University of Vermont, Research Center for Children, Youth, \& Families; 2001. 
73. Reynolds CR, Kamphaus RW. Behavior Assessment System for Children-Second Edition (BASC-2). Bloomington: Pearson; 2004.

74. Streissguth AP, Barr HM, Sampson PD, Parrish-Johnson JC, Kirchner GL, Martin DC. Attention, distraction and reaction time at age 7 years and prenatal alcohol exposure. Neurobehav Toxicol Teratol. 1986;8(6):717-25.

75. Burden MJ, Jacobson SW, Sokol RJ, Jacobson JL. Effects of prenatal alcohol exposure on attention and working memory at 7.5 years of age. Alcohol Clin Exp Res. 2005;29(3):443-52.

76. Jacobson SW, Jacobson JL, Sokol RJ. Effects of fetal alcohol exposure on infant reaction time. Alcohol Clin Exp Res. 1994;18(5):1125-32.

77. Nanson JL, Hiscock M. Attention deficits in children exposed to alcohol prenatally. Alcohol Clin Exp Res. 1990;14(5):656-61.

78. Bhatara V, Loudenberg R, Ellis R. Association of attention deficit hyperactivity disorder and gestational alcohol exposure: an exploratory study. J Att Disord. 2006;9(3):515-22.

79. Streissguth AP, Martin DC, Barr HM, Sandman BM, Kirchner GL, Darby BL. Intrauterine alcohol and nicotine exposure: attention and reaction time in 4-year-old children. Dev Psychol. 1984;20(4):533-41.

80. Jacobson JL, Jacobson SW, Sokol RJ, Martier SS, Ager Jr JW, Kaplan-Estrin MG. Teratogenic effects of alcohol on infant development. Alcohol Clin Exp Res. 1993;17(1):174-83.

81. Aragón AS, Coriale G, Fiorentino D, Kalberg WO, Buckley D, Gossage JP, et al. Neuropsychological characteristics of Italian children with fetal alcohol spectrum disorders. Alcohol Clin Exp Res. 2008;32(11):1909-19. doi:10.1111/j.1530-0277.2008. 00775.x.

82. Brown RT, Coles CD, Smith IE, Platzman KA, Silverstein J, Erickson S, et al. Effects of prenatal alcohol exposure at school age. II. Attention and behavior. Neurotoxicol Teratol. 1991;13(4): 369-76.

83. Mattson SN, Riley EP. Parent ratings of behavior in children with heavy prenatal alcohol exposure and IQ-matched controls. Alcohol Clin Exp Res. 2000;24(2):226-31. doi:10.1111/j.15300277.2000.tb04595.x.

84. Nash K, Rovet J, Greenbaum R, Fantus E, Nulman I, Koren G. Identifying the behavioural phenotype in fetal alcohol spectrum disorder: sensitivity, specificity and screening potential. Arch Women Ment Health. 2006;9(4):181-6. doi:10.1007/s00737006-0130-3.

85. Janzen LA, Nanson JL, Block GW. Neuropsychological evaluation of preschoolers with fetal alcohol syndrome. Neurotoxicol Teratol. 1995;17(3):273-9.

86. Conners CK. Conners Continuous Performance Test, Third Edition (CPT-III). Toronto: Multi-Health Systems Inc.; 2014.

87. Greenberg LM. T.O.V.A. continuous performance test manual. Los Alamitos, CA1996.

88. Quotient ADHD System. Pearson.

89. Conners CK. Conners (Conners 3). 3rd ed. Toronto: Multi-Health Systems; 2008.

90. Raldiris TL, Bowers TG, Towsey C. Comparisons of intelligence and behavior in children with fetal alcohol spectrum disorder and ADHD. J Atten Disord. 2014. doi:10.1177/1087054714563792.

91. McGee CL, Bjorkquist OA, Riley EP, Mattson SN. Impaired language performance in young children with heavy prenatal alcohol exposure. Neurotoxicol Teratol. 2009;31(2):71-5. doi:10.1016/j. ntt.2008.09.004.

92. Church MW, Eldis F, Blakley BW, Bawle EV. Hearing, language, speech, vestibular, and dentofacial disorders in fetal alcohol syndrome. Alcohol Clin Exp Res. 1997;21(2):227-37.

93. Becker M, Warr-Leeper GA, Leeper Jr HA. Fetal alcohol syndrome: a description of oral motor, articulatory, short-term memory, grammatical, and semantic abilities. J Commun Disord. 1990;23(2):97-124. doi:10.1016/0021-9924.90.90016-R.

94. Sparrow SS, Balla DA, Cicchetti DV. Vineland Adaptive Behavior Scales: Survey Form Manual. Interview ed. Circle Pines: American Guidance Service; 1984.

95. Crocker N, Vaurio L, Riley EP, Mattson SN. Comparison of adaptive behavior in children with heavy prenatal alcohol exposure or attention-deficit/hyperactivity disorder. Alcohol Clin Exp Res. 2009;33(11):2015-23. doi:10.1111/j.1530-0277.2009.01040.x.

96. LaDue RA, Streissguth AP, Randels SP. Clinical considerations pertaining to adolescents and adults with fetal alcohol syndrome. In: Sonderegger TB, editor. Perinatal Substance Abuse: Research Findings and Clinical Implications. The Johns Hopkins series in environmental toxicology. Baltimore: The Johns Hopkins University Press; 1992. p. 104-31.

97. Wallace G, Hammill DD. Comprehensive Receptive and Expressive Vocabulary Test-Third Edition (CREVT-3). Austin: Pro-Ed Inc.; 2013.

98. Wiig EH, Semel E, Secord WA. Clinical Evaluation of Language Fundamentals-Fifth Edition (CELF-5). San Antonio: Pearson; 2013.

99. Wiig EH, Secord WA, Semel E. Clinical Evaluation of Language Fundamentals-Preschool-Second Edition (CELF-Preschool-2). San Antonio: The Psychological Corporation; 2004.

100. Sparrow SS, Cicchetti DV, Balla DA. Vineland Adaptive Behavior Scales: Survey Forms Manual. 2nd ed. Circle Pines: AGS Publishing; 2005.

101. Harrison P, Oakland T. Adaptive Behavior Assessment System, Third Edition (ABAS-3). Torrance: Western Psychological Services; 2015.

102. Coggins TE, Olswang LB, Carmichael Olson H, Timler GR. On becoming socially competent communicators: the challenge for children with fetal alcohol exposure. Int Rev Res Mental Retard. 2003;27:121-50. doi:10.1016/S0074-7750(03)27004-X.

103. Coggins TE, Timler GR, Olswang LB. A state of double jeopardy: impact of prenatal alcohol exposure and adverse environments on the social communicative abilities of school-age children with fetal alcohol spectrum disorder. Lang Speech Hear Serv Sch. 2007;38(2):117-27.

104. Timler GR, Olswang LB, Coggins TE. "Do I know what I need to do?" A social communication intervention for children with complex clinical profiles. Lang Speech Hear Serv Sch. 2005;36(1):7385. doi:10.1044/0161-1461(2005/007).

105. Thorne JC, Coggins TE, Carmichael Olson H, Astley SJ. Exploring the utility of narrative analysis in diagnostic decision making: picture-bound reference, elaboration, and fetal alcohol spectrum disorders. J Speech Lang Hear Res. 2007;50(2):459-74.

106. Thomas SE, Kelly SJ, Mattson SN, Riley EP. Comparison of social abilities of children with fetal alcohol syndrome to those of children with similar IQ scores and normal controls. Alcohol Clin Exp Res. 1998;22(2):528-33. doi:10.1111/j.1530-0277. 1998.tb03684.x.

107. Whaley SE, O'Connor MJ, Gunderson B. Comparison of the adaptive functioning of children prenatally exposed to alcohol to a nonexposed clinical sample. Alcohol Clin Exp Res. 2001;25(7): 1018-24. doi:10.1111/j.1530-0277.2001.tb02311.x.

108. Rutter M, Bailey A, Berument SK, Lord C, Pickles A. Social Communication Questionnaire (SCQ). Lifetime version. Los Angeles: Western Psychological Services; 2003.

109. Bishop S, Gahagan S, Lord C. Re-examining the core features of autism: a comparison of autism spectrum disorder and fetal alcohol spectrum disorder. J Child Psy Psychia. 2007;48(11):1111-21. doi:10.1111/j.1469-7610.2007.01782.x.

110. Fagerlund Å, Autti-Rämö I, Kalland M, Santtila P, Hoyme HE, Mattson SN, et al. Adaptive behaviour in children and adolescents with foetal alcohol spectrum disorders: a comparison with specific 
learning disability and typical development. Eur Child Adolesc Psychiatry. 2012;21(4):221-31. doi:10.1007/s00787-012-0256-y.

111. Streissguth AP, Barr HM, Martin DC, Herman CS. Effects of maternal alcohol, nicotine, and caffeine use during pregnancy on infant mental and motor development at eight months. Alcohol Clin Exp Res. 1980;4(2):152-64.

112. Autti-Rämö I, Granström M-L. The psychomotor development during the first year of life of infants exposed to intrauterine alcohol of various duration. Neuropediatrics. 1991;22:59-64.

113. Kooistra L, Ramage B, Crawford S, Cantell M, Wormsbecker S, Gibbard B, et al. Can attention deficit hyperactivity disorder and fetal alcohol spectrum disorder be differentiated by motor and balance deficits? Hum Mov Sci. 2009;28(4):529-42. doi:10. 1016/j.humov.2009.01.007.
114. Doney R, Lucas BR, Jones T, Howat P, Sauer K, Elliott EJ. Fine motor skills in children with prenatal alcohol exposure or fetal alcohol spectrum disorder. J Dev Behav Pediatr. 2014;35(9): 598-609. doi:10.1097/DBP.0000000000000107.

115. Mattson SN, Roesch SC, Fagerlund $\AA$, Autti-Rämö I, Jones KL, May PA, et al. Toward a neurobehavioral profile of fetal alcohol spectrum disorders. Alcohol Clin Exp Res. 2010;34(9):1640-50. doi:10.1111/j.1530-0277.2010.01250.x.

116. Kalberg WO, Provost B, Tollison SJ, Tabachnick BG, Robinson LK, Hoyme HE, et al. Comparison of motor delays in young children with fetal alcohol syndrome to those with prenatal alcohol exposure and with no prenatal alcohol exposure. Alcohol Clin Exp Res. 2006;30(12):2037-45.

117. Folio MR, Fewell RR. Peabody Developmental Motor Scales Examiner's Manual. 2nd ed. Austin: Pro-Ed; 2000. 